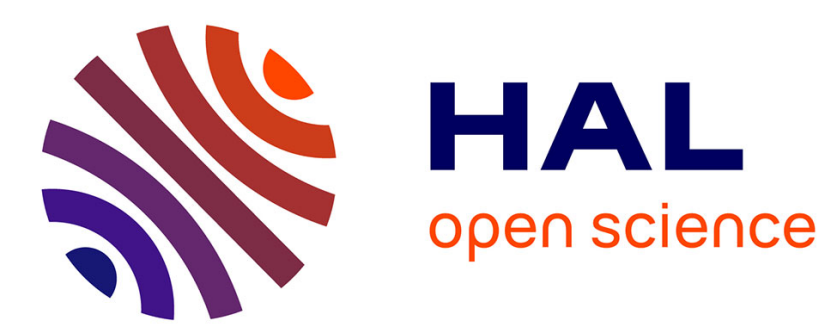

\title{
Incremental Micromechanical Modelling of the Transformation Induced Plasticity
}

J. Diani, M. Berveiller, H. Sabar

\section{To cite this version:}

J. Diani, M. Berveiller, H. Sabar. Incremental Micromechanical Modelling of the Transformation Induced Plasticity. Journal de Physique IV Proceedings, 1996, 06 (C1), pp.C1-419-C1-427. 10.1051/jp4:1996140 . jpa-00254172

\section{HAL Id: jpa-00254172 https://hal.science/jpa-00254172}

Submitted on 1 Jan 1996

HAL is a multi-disciplinary open access archive for the deposit and dissemination of scientific research documents, whether they are published or not. The documents may come from teaching and research institutions in France or abroad, or from public or private research centers.
L'archive ouverte pluridisciplinaire HAL, est destinée au dépôt et à la diffusion de documents scientifiques de niveau recherche, publiés ou non, émanant des établissements d'enseignement et de recherche français ou étrangers, des laboratoires publics ou privés. 


\title{
Incremental Micromechanical Modelling of the Transformation Induced Plasticity
}

\author{
J.M. Diani, M. Berveiller* and H. Sabar* \\ Centre de Recherches et d'Etudes d'Arcueil, 94114 Arcueil, France \\ * LPMM, ISGMP, Ile du Saulcy, 57045 Metz, France
}

\begin{abstract}
Classical plasticity (dislocation motion) coupled to solid-solid phase transformation (lattice changes) is the primary cause of the special behaviour of TRIP (TRansformation Induced Plasticity) steels. Internal stresses associated with the phase change induces in fact a large additional plastic flow inside austenite and martensite. We propose a kinematic description of such a phenomenon based on a local decomposition of strain rate into an elastoplastic part and a given lattice inelastic strain rate field. Based on this description and using usual Green functions method, a concentration tensor for the total strain rate field is obtained. The self consistent approximation allows to determine the behavior of the equivalent material and the equivalent transformation strain rate.
\end{abstract}

\section{INTRODUCTION}

In the case of TRIP steel the determination of the global behavior during phase transformation is complicated since the response of the material to an external thermomechanical loading is not only due to a large scale orientation of the transformation strain of martensite [1] (Bain strain) but also to a large scale orientation of plastic flow in austenite [2] and martensite due to irreversible strains at the microscopic scale. The aim of this paper is to give an analysis of this point based on a kinematic description at a microscopic level that allows to propose a micromechanical model of the phenomenon.

In this paper one does not deal with the two following questions arising naturally in the case of martensitic transformation :

- to express volume fraction $f$ of martensite versus loading (controlling) external parameters (stress $\Sigma$ or strain $E$ and temperature $T$ ):

$$
\dot{f}=() \dot{\Sigma}+() \dot{T}
$$

- to give the mean value of the transformation strain (or strain rate) over all the different martensite variants :

$$
\overline{\dot{\varepsilon}}^{B}(t)=\frac{1}{V} \int_{V} \dot{\varepsilon}^{B}(r, t) d s=() \dot{\Sigma}+() \dot{T}
$$

that is to solve the problem posed by [1].

In the present work $\dot{f}$ and $\overline{\dot{\varepsilon}}^{B}$ are assumed to be known. The aim of this paper is to find a relation between $\dot{E}, \dot{\Sigma}$ and $\overline{\dot{\varepsilon}}^{B}$ such as :

$$
\dot{E}=() \dot{\Sigma}+() \bar{\varepsilon}^{B}=() \dot{\Sigma}+\dot{E}^{T R}
$$


that can be seen as an overall behavior law for TRIP steels. $\dot{E}^{T R}=() \overline{\dot{\varepsilon}}^{B}$ is defined here as the macroscopic transformation strain rate.

The kinematics and transformation kinetics is studied in paragraph $\mathbf{2}$ and leads to a precise definition of $\overline{\dot{\varepsilon}}^{B}$ versus a microscopic description of the structure. A micromechanical model is then established in paragraph 3 giving explicit formulae for equation (3).

\section{KINEMATICS AND PHASE TRANSFORMATION KINETICS}

In the case of martensitic phase transformation in ductile metals one has to take into account several type of deformations or distortions (Fig. 1).

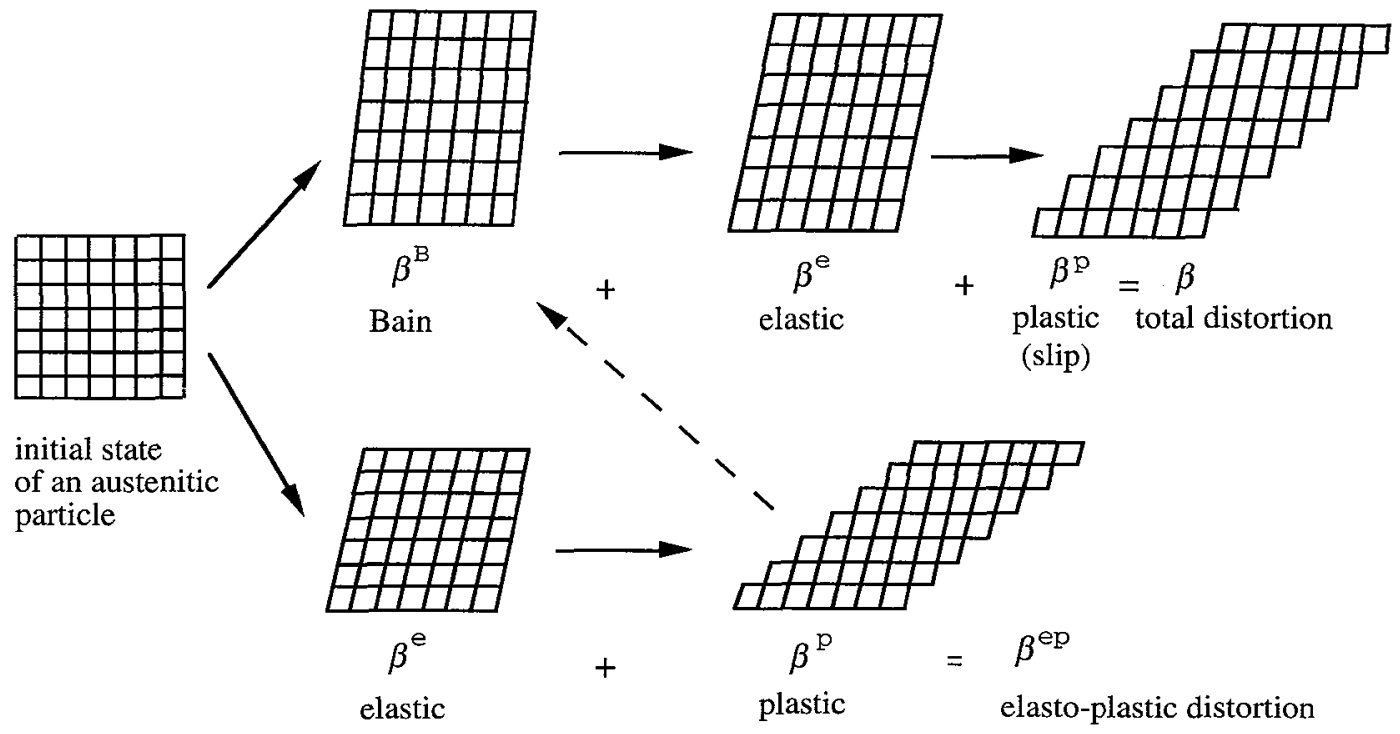

Figure 1: Schematic decomposition of the total distortion into elastic, lattice inelastic (or Bain) and lattice invariant or plastic parts for a particle during martensitic phase transformation and a particle without transformation.

- Firstly, austenite and martensite are supposed to be elastic so that an elastic distortion of the two lattices has to be introduced.

- Next, due to the change of lattice during phase transformation, an inelastic (in the sense of stress free strain (Eshelby [3], Kröner [4]) lattice distortion $\beta^{B}(r, t)$ describes the transformation of the austenitic to the martensitic lattice (several variants may be reached from a given austenitic lattice). $\beta^{B}(r, t)$ is an inelastic primary field which corresponds to an observable state variable; it is usually given by the metallurgical observations and can be measured, for example, by X-rays.

$\beta^{B}(r, t)$ is treated here as a small strain and can therefore be written as $\beta^{B}(r, t)=\varepsilon^{B}(r, t)+w^{B}(r, t)$, where $\varepsilon^{B}(r, t)$ is the symmetric part of $\beta^{B}(r, t)$ (the Bain strain) and $w^{B}(r, t)$ the antisymmetric part.

- For ductile metals, an inelastic distortion may occur in austenite and martensite due to plastic flow, associated with creation, annihilation and movement of dislocations (slip). This inelastic lattice invariant distortion $\varepsilon^{p}(r, t)$ is also used in the case of the small strain hypothesis.

Using the previous local kinematic description, one considers now a macroscopic element of matter (volume $V$ ) containing two phases (global description). Under a thermomechanical loading, one 
part (previously austenite) has been transformed into martensite, i.e. a lattice transformation has occurred, defined locally (and completely) by the deformation $\varepsilon^{B}(r, t)$ and rotation $w^{B}(r, t)$.

The martensite volume $V_{m}$ is generally made of a lot of separated elementary volumes $V_{m}^{I} \quad(I=1, \ldots, N)$ which can be seen as martensite laths or variants of different orientations (lattice and shape) inside whose $\beta^{B}(r, t)$ is uniform and constant. The transformation proceeds with the growth of such existing domains and the appearance of new ones (Fig. 2).

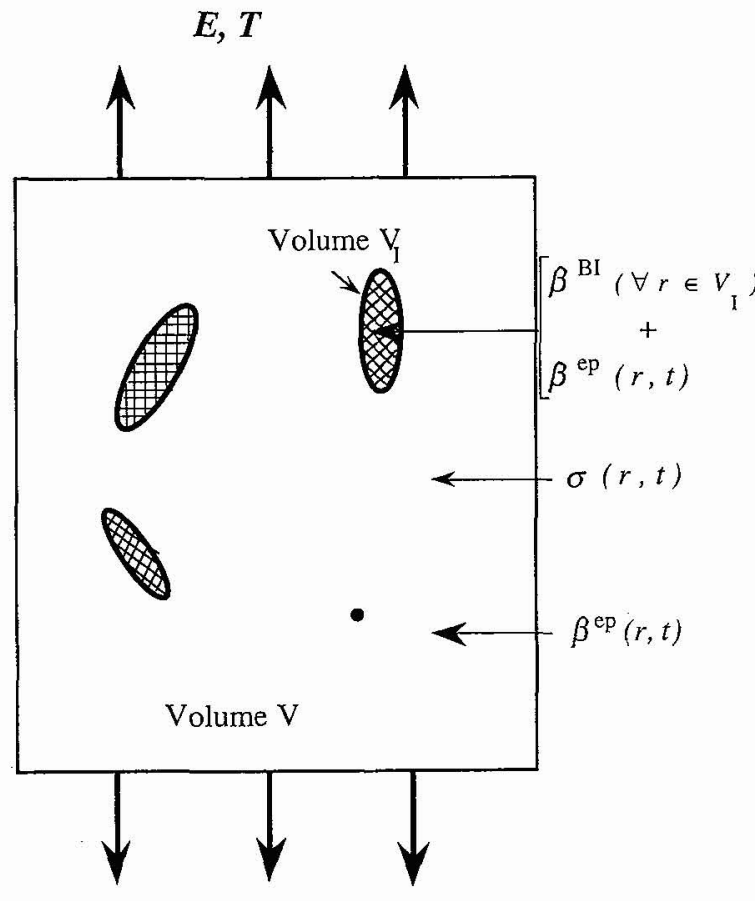

Current state defined globaly by $\mathbf{E}, \mathbf{T}$

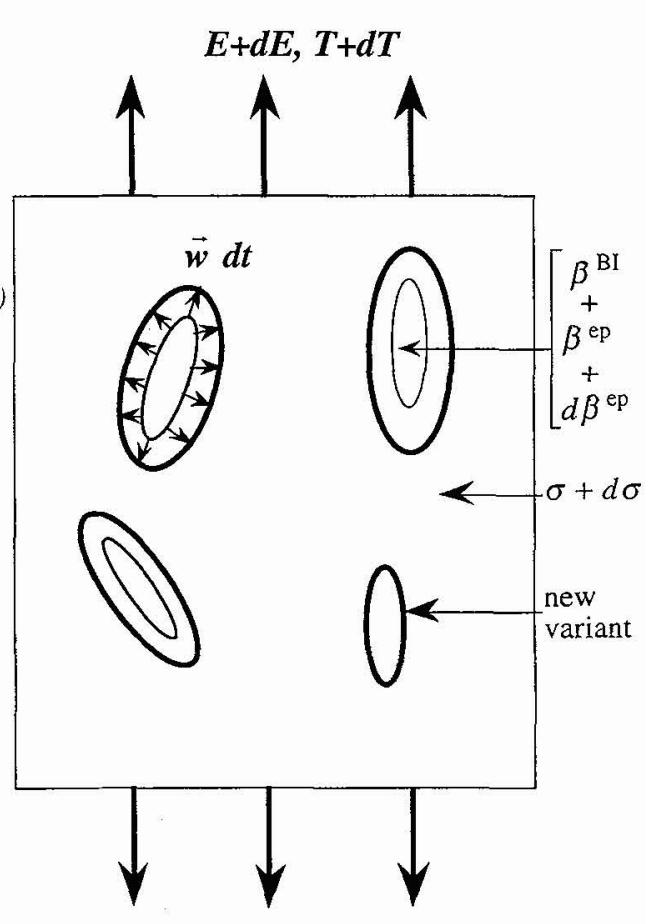

After loading by $d E, d T$

Figure 2 : Schematic representation of the evolution of the transformation and related fields.

$E, d E$ : overall imposed strain and strain increment.

$T, d T:$ imposed temperature and temperature change.

$\vec{W}$ : velocity of the interface between austenite and martensite.

The inelastic part of the lattice distortion (i.e. the Bain distortion) can therefore be written as :

$$
\beta_{i j}^{B}(r, t)=\sum_{i=1}^{N} \beta_{i j}^{B_{I}} \theta^{t}(r, t)
$$

where $\theta^{l}(r, t)$ is defined by:

$$
\theta^{\prime}(r, t)=\left\{\begin{array}{lll}
0 & \text { if } & r \notin V_{m}^{I} \\
1 & \text { if } & r \in V_{m}^{I}
\end{array}\right.
$$


and $\beta^{B_{I}}$ is the Bain inelastic distortion in the Ith volume and is assumed to be uniform and constant.

The transformation is driven here by a thermomechanical loading. The evolution is followed by a geometric extension of the transformed zone due to the velocity field $\vec{W}^{I}(r, t)$ of the domains $\left(V_{m}^{I}\right)$ boundaries $S^{I}$, those velocities being of course different from the particle ones.

The transformation field $\beta^{B}(r, t)$, which is difficult to get in general, must be obtained from a thermodynamical analysis that is not presented here, and is therefore supposed to be known, at least partially. As a first order approximation, the mean value of the $\beta^{B}(r, t)$ field may be used for describing the transformation rate. Moreover only those mean values are observable using global magnetometric or $\mathrm{X}$-ray diffraction measurements.

One defines therefore the mean transformation strain rate in the volume $V$ by :

$$
\overline{\dot{\varepsilon}}^{B}(t)=\frac{l}{V} \int_{V} \dot{\varepsilon}^{B}(r, t) d V
$$

that can be written as (see [5]) :

where

$$
\overline{\dot{\varepsilon}}_{i j}^{B}(t)=\bar{\varepsilon}_{i j}^{B} \dot{f}(t)
$$

$$
\dot{f}(t)=\frac{\dot{V}_{m}}{V}=\sum_{I=1}^{N} \dot{f}^{I}(t)
$$

is the total volume fraction variation of martensite, $f^{I}(t)$ is the volume fraction of each lath or variant defined as :

$$
f^{I}(t)=\frac{1}{V} \int_{V} \theta^{I}(r, t) d V
$$

and $\bar{\varepsilon}^{B}(t)=\frac{\sum_{I=1}^{N} \varepsilon^{B_{I}} \dot{f}^{I}(t)}{\sum_{I=1}^{N} \dot{f}^{I}(t)}$ defines the mean transformation strain of volume $V$ occurring at time $t$. The two variables $\dot{f}$ and $\bar{\varepsilon}^{B}$ are commonly measured by the methods described previously.

\section{MICROMECHANICAL MODEL}

\subsection{The TRIP effect}

In the case of transformation induced by cooling $(\dot{T}<0)$ under constant applied stress $(\dot{\Sigma}=0)$, the macroscopic registered strain rate $\dot{E}^{T R}$ is, in general, higher than $\overline{\dot{\varepsilon}}^{B}$. The field $\dot{\varepsilon}^{B}(r, t)$ is in fact strongly incompatible, inducing internal stresses and elastoplastic flow so that the total strain rate field becomes compatible ; plasticity appears in the "weakest phase" (with the lowest yield strength, here the austenite) but also in general in the martensite. The previous discussion can finally be resumed in $\dot{E}^{T R}>\overline{\dot{\varepsilon}}^{B}$ which is a generalization of the TRIP effect.

This point can be underlined using the works of Kröner [6], Hill and Rice [7] and Mandel [8] : the particle velocity gradient $v_{i, j}(r, t)$ can be divided into an elasto-plastic part $\dot{\beta}^{e p}(r, t)$ and the part corresponding to the phase transformation $\dot{\beta}^{B}(r, t)$ as :

$$
v_{i, j}(r, t)=\dot{\beta}_{i j}^{e p}(r, t)+\dot{\beta}_{i j}^{B}(r, t)
$$

where the elasto-plastic part added classically in elastic and plastic strain rate, such as: $\dot{\beta}_{i j}^{e p}(r, t)=\dot{\beta}_{i j}^{e}(r, t)+\dot{\beta}_{i j}^{p}(r, t)$. The general thermomechanical loading conditions on volume $V$ are : 
- an imposed stress rate : $\dot{\Sigma}_{i j}(t) n_{j}$,

- a uniform temperature change $\dot{T}(t)$

inducing a transformation rate field $\dot{\varepsilon}^{B}(r, t)$, an elasto-plastic strain rate field $\dot{\varepsilon}^{e p}(r, t)$ and a stress rate field $\dot{\sigma}(r, t)$. In the following, the dependence on kinematic time $\mathbf{t}$ shall be omitted to get simplier equations. Using Hill lemma and conditions one finds that the local stress rate field and the macroscopic stress rate are related by:

$$
\dot{\Sigma}_{i j}=\bar{\sigma}_{i j}=\frac{1}{V} \int_{V} \dot{\sigma}_{i j}(r) d V
$$

and the resulting macroscopic strain rate is given by :

$$
\dot{E}_{i j}=\frac{1}{V} \int_{V} \dot{\varepsilon}_{i j}(r) d V=\frac{I}{V} \int_{V}\left(\dot{\varepsilon}_{i j}^{e p}(r)+\dot{\varepsilon}_{i j}^{B}(r)\right) d V
$$

Substituting in (12) the tangent local behavior $\dot{\varepsilon}_{i j}^{e p}(r)=m_{i j k l}(r) \dot{\sigma}_{k l}(r)$, one gets :

$$
\dot{E}_{i j}=\frac{1}{V} \int_{V} m_{i j k l}(r) \dot{\sigma}_{k l}(r) d V+\frac{1}{V} \int_{V} \dot{\varepsilon}_{i j}^{B}(r) d V
$$

Due to the internal stresses associated with the transformation rate $\dot{\varepsilon}^{B}(r)$, the first term is not equal to zero even if $\dot{\Sigma}_{i j}=O$ so that $\dot{E} \neq \overline{\dot{\varepsilon}}^{B}$. On the other hand, in the particular and restrictive case of an homogeneous material ( $m_{i j k l}$ space independant), (13) becomes :

wich reduces in :

$$
\dot{E}_{i j}=m_{i j k l} \frac{l}{V} \int_{V} \dot{\sigma}_{k l}(r) d V+\frac{1}{V} \int_{V} \dot{\varepsilon}_{i j}^{B}(r) d V
$$

$$
\dot{E}=\overline{\dot{\varepsilon}}^{B}
$$

under macroscopic constant applied stresses. In that situation $\left(\Sigma_{i j}=0\right)$, the macroscopic strain rate is equal to the macroscopic transformation strain rate (see introduction), so that (15) becomes

$$
\dot{E}_{i j}^{T R}=\dot{f} \bar{\varepsilon}_{i j}^{B}
$$

This is the most famous case where the macroscopic transformation strain rate reduces to the mean value of the local Bain strain rate over the volume $\mathrm{V}$.

In general the behavior of the different phases is strongly heterogeneous ; the TRIP effect $\left(\dot{E}^{T R}>\overline{\dot{\varepsilon}}^{B}\right.$ ) corresponds then to that described by Greenwood and Johnson who added an extra term to $\overline{\bar{\varepsilon}}^{B}$ which corresponds to a plastic flow in the two phases to get the macroscopic transformation strain rate.

This extra term is of course depending on the applied stresses. It is also the case for the mean transformation strain rate $\overline{\dot{\varepsilon}}^{B}$ which is due to formation of domains or variants oriented by the applied stresses (Magee mechanism). This last question has been solved - Patoor and al. [9], [10] and Sun and al. [11] - in the case of shape memory alloys for which the medium can reasonably be taken as elastic and homogeneous.

In the present work the problem posed by Greenwood and Johnson (first problem) will be treated which consists in determining the global behavior

$$
\dot{\Sigma}=L\left(\dot{E}-\dot{E}^{T R}\right)
$$


of a solid under a mechanical loading (with boundary conditions on $\partial V$ given by $v_{i}=\left(\dot{E}_{i j}+\dot{\Omega}_{i j}\right) x_{j}$ and with a given and known transformation strain rate $\dot{\beta}^{B}(r, t)$. The constitutive equation of each phase is supposed to be known and given by the usual elasto-plastic constitutive relation : $\dot{\sigma}(r)=l(r) \dot{\varepsilon}^{e p}(r)$. Finally the process presented here uses and extends the micromechanical methods developed by Kröner [12] and by Berveiller and Zaoui [13] and Lipinski and Berveiller [14] to model the plastic behavior of polycrystalline metals.

\subsection{General micromechanical model}

The problem governing equations are :

- quasi-static equilibrium (no volumic forces) :

- kinematic relations :

$$
\dot{\sigma}_{i j, j}(r)=0
$$

- constitutive equations :

$$
v_{i, j}(r)=\dot{\beta}_{i j}^{e p}(r)+\dot{\beta}_{i j}^{B}(r)
$$

$$
\dot{\sigma}_{i j}(r)=l_{i j k l}(r) \dot{\beta}_{k l}^{e p}(r)
$$

The particle velocity $v_{i}$ is the unknown variable so that one finds classically using (17), (18) and (19):

$$
\left[l_{i j k l}(r)\left(v_{k, l}(r)-\dot{\varepsilon}_{k l}^{B}(r)\right)\right]_{, j}=0
$$

As for heterogeneous elastic (see Dederichs and Zeller [15], Kröner [4] and Willis [16] or elastoplastic media (see Berveiller and Zaoui [13] and Lipinski and Berveiller [14], one introduces a reference homogeneous medium with tangent modulus $L^{o}$ which undergoes an uniform transformation strain rate of value $\dot{E}^{r R^{o}}$ such as :

and

$$
l(r)=L^{o}+\delta l^{o}(r)
$$

$$
\dot{\varepsilon}^{B}(r)=\dot{E}^{T R^{o}}+\delta \dot{\varepsilon}^{B o}(r)
$$

Using the Green tensor $G^{o}$ of an homogeneous infinite medium of tangent modulus $L^{o}$ defined by :

$$
L_{i j k l}^{o} G_{j n, i k}^{o}\left(r-r^{\prime}\right)+\delta_{l n} \delta\left(r-r^{\prime}\right)=0
$$

the strain rate $\dot{\varepsilon}=\frac{1}{2}\left(\nabla \vec{v}+\nabla^{T} \vec{v}\right)$ is given by :

$$
\dot{\varepsilon}_{i j}(r)=\dot{E}_{i j}+\int_{V^{\prime}} \Gamma_{i j k l}^{o}\left(r-r^{\prime}\right)\left[\delta l_{k l m n}^{o}\left(r^{\prime}\right) \dot{\varepsilon}_{m n}^{e p}\left(r^{\prime}\right)-L_{k i m n}^{o} \delta \dot{\varepsilon}_{m n}^{B^{a}}\left(r^{\prime}\right)\right] d V^{\prime}
$$

where $\Gamma^{\circ}$ represents the modified Green tensor of infinite medium $L^{o}$ introduced by Kröner [17] for elasticity and defined by :

$$
\Gamma_{i j k l}^{o}=\frac{1}{2}\left[G_{i k, j l}^{o}+G_{j k, i l}^{o}\right]
$$

The L.S.D type equation (23) has to be solved in order to obtain the overall behavior. One may find in [5] the usual TAYLOR-LIN approximation for (23). In the following we deal with a more refined selfconsistent approximation. 


\subsection{Self consistent approximation in the case of perfect disordered media for integral equation (23)}

The self consistent approach has its great interest in the modified Green tensor properties which can be divided for any infinite homogeneous medium with tangent moduli $L^{o}$ into a local (l) part $\Gamma^{o l}$ and a non-local (nl) part $\Gamma^{o n l}[15]$ such as :

$$
\Gamma^{o}(r)=\Gamma^{o l} \delta(r)+\Gamma^{o n l}(r)
$$

Replacing (25) in (23) one gets :

$$
\begin{gathered}
\dot{\varepsilon}_{i j}(r)=\dot{E}_{i j}+\Gamma_{i j k l}^{o l}\left[\delta l_{k l m n}^{o}(r) \dot{\varepsilon}_{m n}^{e p}(r)-L_{k l m n}^{o} \delta \dot{\varepsilon}_{m n}^{B^{o}}(r)\right]+ \\
\int_{V^{\prime}} \Gamma_{i j k l}^{o n l}\left(r-r^{\prime}\right)\left[\delta l_{k l m n}^{o}\left(r^{\prime}\right) \dot{\varepsilon}_{m n}^{e p}\left(r^{\prime}\right)-L_{k l m n}^{o} \delta \dot{\varepsilon}_{m n}^{B^{o}}\left(r^{\prime}\right)\right] d v^{\prime}
\end{gathered}
$$

where only the integral term is difficult to calculate due to important and stochastic fluctuations of the field:

$$
F_{k l}\left(r^{\prime}\right)=\delta l_{k m n}^{o}\left(r^{\prime}\right) \dot{\varepsilon}_{m n}^{e p}\left(r^{\prime}\right)-L_{k l m n}^{o} \delta \dot{\varepsilon}_{m n}^{B^{o}}\left(r^{\prime}\right)
$$

The idea of the self consistent scheme, which was initially constructed for heterogeneous media without transformation ( $L_{i j k t}^{o} \delta \dot{\varepsilon}_{k i}^{B^{v}}=0$ ), consists in choosing the reference media ( $L^{o}$ and $\dot{E}^{T R^{o}}$ ) so that the mean value of $F_{k l}$ over $V$ is zero which leads to neglect $\int_{V} \Gamma_{i j k l}^{o n l}(r-r,) F_{k l}(r) d v$, in (26) (see Kröner [18]). This choice can be written as :

$$
\int_{V^{\prime}}\left[\left(l_{i j k l}\left(r^{\prime}\right)-L_{i j k l}^{o}\right) \dot{\varepsilon}_{k l}^{e p}\left(r^{\prime}\right)-L_{i k k l}^{o}\left(\dot{\varepsilon}_{k t}^{B}\left(r^{\prime}\right)-\dot{E}_{k l}^{T R^{o}}\right)\right] d \nu^{\prime}=0
$$

which becomes, using (18) and (19) :

$$
\dot{\Sigma}_{i j}=L_{i j k l}^{o}\left(\dot{E}_{k l}-\dot{E}_{k l}^{T R^{o}}\right)
$$

that is the macroscopic constitutive equation assumed in introduction at the end of paragraph 2 , using $L^{o}=L$ and $\dot{E}^{T R^{0}}=\dot{E}^{T R}$.

(26) becomes then :

where :

$$
\dot{\varepsilon}_{i j}(r)=\dot{E}_{i j}+\Gamma_{i j k l}^{l}\left[\delta l_{k l m n}(r) \dot{\varepsilon}_{m n}^{e p}(r)-L_{k l m n} \delta \dot{\varepsilon}_{m n}^{B}(r)\right]
$$

and

$$
\delta l(r)=l(r)-L
$$

$$
\delta \dot{\varepsilon}^{B}(r)=\dot{\varepsilon}^{B}(r)-\dot{E}^{T R}
$$

are the fluctuations of the fields $l(r)$ and $\dot{\varepsilon}^{B}(r)$ regarding the effective medium $\left(L, \dot{E}^{T R}\right)$ and where $\Gamma^{l}$ is the local part for the modified Green tensor of the effective medium.

(30) should be rewritten as :

defining :

$$
\dot{\varepsilon}_{i j}(r)=A_{i j k l}(r) \dot{E}_{k l}+B_{i j}(r)
$$

and

$$
A_{i j k l}(r)=\left[I_{i j k l}-\Gamma_{i j m n}^{l} \delta l_{m n k l}(r)\right]^{-1}
$$

$$
B_{i j}(r)=A_{i ; k l}(r) \Gamma_{k l m n}^{l}\left[L_{m n p q} \dot{E}_{p q}^{T R}-l_{\text {manpq }}(r) \dot{\varepsilon}_{p q}^{B}(r)\right]
$$


$I_{i j k l}$ is the fourth order unit tensor. The definition of $\dot{E}(12)$ leads easily to :

and

$$
\overline{A_{i j k l}}=\frac{1}{V} \int_{V} A_{i j k l}(r) d V=I_{i j k l}
$$

$$
\bar{B}_{i j}=\frac{I}{V} \int_{V} B_{i j} d V=0
$$

(34) gives the effective tangent modulus $L_{i j k l}$. This resolution leads to the same result as for elastoplastic media without transformation (with given volume fraction of martensite $f$ ). On the other hand, (35) leads to the macroscopic transformation strain rate $\dot{E}^{T R}$ :

$$
\dot{E}^{T R}=L^{-l} \Gamma^{l^{-1}} \overline{A(r) \Gamma^{l} l(r) \dot{\varepsilon}^{B}(r)}
$$

\section{APPLICATION AND CONCLUSION}

In the case of a two phase (austenite $(a)$ that transforms in martensite $(m)$ ) incompressible isotropic material, simple relations can be derived from the previous paragraph (see [5] for details). The effective tangent shear modulus $\mu$ is given by :

$$
f \frac{5 \mu}{3 \mu+2 \mu^{m}}+(1-f) \frac{5 \mu}{3 \mu+2 \mu^{a}}=1
$$

which is a simplification of (34), where $\mu^{a}$ and $\mu^{m}$ are the tangent shear moduli in austenite and martensite (which are supposed to be known) and $f$ the volume fraction of martensite. (35) becomes :

$$
\dot{E}_{i j}^{T R}=\frac{5 \mu^{m}}{3 \mu+2 \mu^{m}} \dot{f} \bar{\varepsilon}_{i j}^{B}
$$

Equations (3), (37) and (38) allow to determine the overall behavior in various situations where $\mu^{a}, \mu^{m}$, $\bar{\varepsilon}^{B}$ and $\dot{f}$ are given (see [5]).

Based on the same scheme than the secant one [19] a general tangent modelling is then obtained for the transformation induced plasticity in steels. It leads to enlightening simple formula in the case of incompressible isotropic two-phase media which are valid either for stress assisted martensite [5] and strain-induced martensite in the case, for example, of the "dynamic softening" [20].

The effect of the isotropic and deviatoric parts of the Bain transformation are not coupled at this step but should be coupled in the future considering the transformation morphology. 


\section{REFERENCES}

[1] Magee C.L. , Transformation kinetics, microplasticity and aging of martensite in FE31Ni (Ph. D. thesis Carnegie Inst. Technology, Pittsburg PA, 1966).

[2] Greenwood G.W. and Johnson R.H., Proc. Roy. Soc. A283 (1965) pp. 403-418

[3] J. D. Eshelby, Progress in Solids Mechanics. Ed. I.N SNEDDON and R. HILL, vol. 2, Amsterdam, North-Holland, pp. 87 (1961).

[4] E. Kröner, In : ModelingSmall Deformation of Polycrystals, Ed. J. Gittus and J. Zarka, Elsevier Applied Science, London, 229 (1986).

[5] J.M. Diani, M. Berveiller and H. Sabar, to be published in Int. J. Engng. Sci.

[6] E. Kröner, Kontinuums Theorie der Versetzungen und Eigenspannungen, Springer Verlag (1958).

[7] R. Hill and J. R. Rice, J. Mech. Phys. Solids 20, 401 (1972).

[8] J. Mandel, Int. J. Solids and Structures 17, 873 (1981).

[9] E. Patoor, A. Eberhardt and M. Berveiller, Acta Metall. 35, 2779 (1987).

[10] E. Patoor, A. Eberhardt and M. Berveiller, Arch. Mech. 40, 775 (1988).

[11] Q. P. Sun, K. C. Hwang and S. W. Yu, J. Mech. Phys. Solids 39, 507 (1991).

[12] E. Kröner, Z. Physik 151, 504 (1958).

[13] M. Berveiller and A. Zaoui, J. Eng. Mat. Tech. 106, 295 (1984).

[14] P. Lipinski and M. Berveiller, Int. J. Plasticity 5, 149 (1989).

[15] P. H. Dederichs and R. Zeller, Z. Physik 259, 103 (1973).

[16]J. R. Willis, J. Mech. Phys. Solids 25, 185 (1977).

[17] E. Kröner, In : Micromechanics and inhomogeneity,Weng, G. J., Taya, M. and Abe, M. Ed. Springer Verlag, 197 (1989).

[18] E. Kröner, J. Mech. Phys. Solids 21, 9 (1977).

[19] J.M. Diani, M. Berveiller and H. Sabar, Materials Science Forum 123-125, 101.(1993).

[20] E. Gautier, X.M. Zhang and A. Simon, Proc. 2nd Int. Conf. Residual Stresses ICRS2, eds.G. Beck, S. Denis and A. SIMON, Elsevier, London, 777-783, (1989). 